\title{
Case report of an intra-abdominal desmoid tumour presenting with bowel perforation
}

\author{
Mushtaq Shah*, Bushra Azam
}

\begin{abstract}
Desmoid tumours are benign tumours originating from the musculoaponeurotic structures of the body. They are mainly composed of collagen. These tumours commonly occur in post-partum women in whom they originate from the rectus abominus and in old surgical incisions. Here we present a case report of a young gentleman who presented with an acute surgical abdomen and subsequently underwent a laparotomy and was found to have an inflammatory mass. Histological analysis of this mass revealed mesenteric fibromatosis (desmoid tumour). I wish to present this interesting case because the patient was neither female nor someone who had previously undergone any surgery. It is rare for mesenteric fibromatosis to present with intestinal perforation and only one case been reported in the literature thus far (1). An important learning point from this case is that rare pathology can manifest itself with common signs and symptoms.
\end{abstract}

CASE

History \& examination

A 33 year old healthy male of Afro-Caribbean origin presented to the emergency department with acute generalised abdominal pain of three hours duration. He had not noticed any recent change in bowel habit, bleeding per rectum or any systemic symptoms. His past medical history was unremarkable. It was of note that there was no family history of inflammatory bowel disease or gastrointestinal malignancy. He was a nonsmoker and did not consume alcohol. On examination he had a heart rate of 110 beats per minute, temperature of $37.2^{\circ} \mathrm{C}$, stable blood pressure but he was cold and clammy. Examination of his abdomen revealed a peritonitic abdomen that was not distended. No mass was palpable and there was no organomegaly. Bowel sounds were absent and rectal exam revealed an empty rectum and no palpable masses.

\section{Investigations}

An erect chest x-ray revealed a pneumoperitoneum.

\footnotetext{
*To whom correspondence should be addressed: Mushtaq Shah 13 Sycamore Avenue, Grimsby, DN33 2AY, U.K.

Email: mushtaqshah@googlemail.com
}

Blood tests revealed a neutrophilia of 25,000 but urea and electrolytes were within normal range.

\section{Treatment}

He was resuscitated with intravenous fluids and an emergency laparotomy was performed. During the laparotomy, a $0.5 \mathrm{~cm}$ perforation was noted in the ileum $25 \mathrm{~cm}$ proximal to the ileo-caecal valve on the mesenteric border. An inflammatory mass measuring 5 $\mathrm{x} 3 \mathrm{~cm}$ was noted directly adjacent to the perforation in the mesentry. $30 \mathrm{~cm}$ of small bowel was resected with a right hemicolectomy, hence removing the mass and the perforation. A side-to-side anastomosis was done.

\section{Post-operative course}

The patient had a swinging pyrexia for a period of one week. No obvious cause of sepsis was identified and it was thought that he may have a subphrenic collection. Therefore a computed tomography (CT) scan was arranged. However, he had a severe reaction to the intravenous contrast medium which resulted in hypotension. Fortunately, the patient made a full recovery. The scan confirmed the suspicion of a subphrenic abscess. The patient underwent a second laparotomy to drain the subphrenic abscess and no masses were seen during this procedure. Histological 
examination of the resected specimen from the first laporotomy revealed mesenteric fibromatosis (desmoid tumour) incompletely excised. The resected right hemicolon did not show evidence of dysplasia, malignancy or polyposis. The estrogen receptor status was not reported. Due to continuing abdominal discomfort on the ward after the second laparotomy, an ultrasound scan was arranged that revealed the following:

A rather ill-defined, fairly ovoid, inhomogeneouslyhypoechoic mass lesion is identified in the lower abdomen (infra- umbilical) para-sagittal plane. It measures about 5 x $3 \times 4.5 \mathrm{~cm}$ in maximum dimensions surrounded by excess peritoneal fat and some bowel loops representing a residual of a previously resected abdominal desmoid.

The pain eventually resolved and after making a good recovery, he was commenced on oral tamoxifen, $20 \mathrm{mg}$ once a day. Although estrogen receptor status was not reported, the patient was commenced on this therapy as it was assumed that most desmoid tumours have estrogen receptors. He was subsequently referred to a specialist in a distant tertiary unit for further evaluation of the residual tumour and for the prospect of further elective surgery.

\section{DISCUSSION}

Desmoid tumours (fibromatosis) are benign fibrous neoplasms originating from the musculo-aponeurotic structures throughout the body. These tumours are locally aggressive. Their treatment is rather difficult as they have a strong tendency to recur. Desmoid tumours can arise from any skeletal muscle but commonly affect the rectus abdominus in post-partum females and in old surgical scars of the abdomen. Our patient did not fit into either category. Desmoids can present in two forms, peripheral or intra-abdominal. Peripheral tumours are smooth, firm and mobile. They are adherent to the surrounding structures. The overlying skin is unaffected (2). Gardner's syndrome and familial adenomatous polyposis coli (FAP) should be suspected in patients with such soft tissue growths (3).

An intra-abdominal desmoid, also known as mesenteric fibromatosis, is the most common solid primary neoplasm of the mesentry. However, it is the least common subtype of fibromatosis. Approximately $80 \%$ of intra-abdominal desmoids involve small bowel mesentry, as was the case with our patient. Involvement of the transverse mesocolon, retroperitoneum, omentum and the ligamentum teres have also been reported $(1,4)$. Intra-abdominal desmoids are usually asymptomatic until their growth and infiltration causes compression of the viscera. This can lead to intestinal obstruction, ischemic bowel secondary to vascular compression and hydronephrosis due to ureteric compression. Bowel perforation is extremely rare and my research has yielded one previous case report (1). Other rare manifestations reported in the literature include deep vein thrombosis, pyrexia of unknown origin, gastrointestinal bleeding and intra-abdominal abscess formation $(5,6)$. The subphrenic abscess that our patient developed is very likely to have been a complication of the initial laparotomy. Histologically, these tumours are composed of collagen that surrounds spindle cells which are poorly circumscribed (Figure 12 ). The cytoplasm is pale with regular nuclei, with neither mitoses nor giant cells. Macrophages, giant cells and lymphocytes are present in the periphery (7).

Exact etiology is uncertain but hormonal factors are implicated as there are estrogen eceptors present in some desmoid tumours. They appear in young women during and after pregnancy (2). They begin to regress

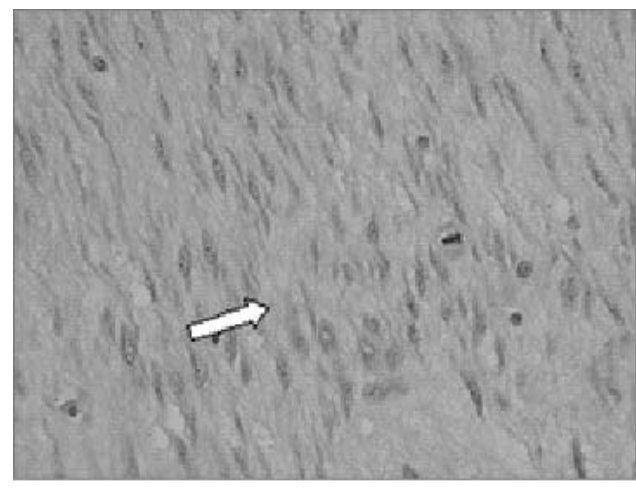

Figure 1. Spindle shaped cells (arrow) (1).

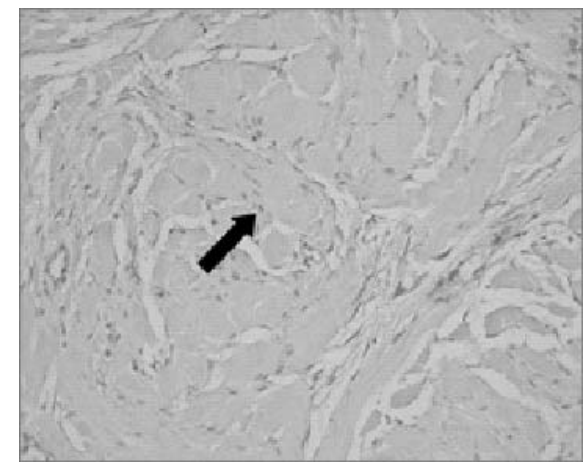

Figure 2. Collagen deposition (arrow) (1).

during menopause and with tamoxifen therapy and even oral contraceptives. The adenomatous polyposis coli (APC) gene located on chromosome 5 is responsible for familial adenomatous polypsois coli (FAP). Biallelic mutations of the APC gene induces desmoid tumour formation, hence the association between these two disorders (8). In our patient, there was no polyposis in the resected right hemi-colon. Trauma has also been suggested as another causative factor but there was no such history in our patient (1). 
Both $\mathrm{CT}$ and magnetic resonance imaging (MRI) are useful in diagnosing and monitoring recurrence. They allow accurate staging and also enable one to delineate accurate anatomy prior to embarking upon major resection. MRI has the advantage of defining the extent of involvement and monitoring post operative recurrence, which can be as high as $70 \%$ (6). These patients should also undergo colonoscopy and examination of the eye to exclude Gardner's syndrome. Colonoscopy may reveal multiple colonic polyps and fundoscopy may demonstrate multiple pigmented lesions affecting the fundus of the eye $(2,3)$.

Complete surgical excision may be the only effective way of providing a cure but complete excision is often impossible and therefore adjuvant treatments have been employed with various degrees of success (1, 2). Other treatment modalities include radiotherapy, if there is recurrence or as primary treatment to avoid radical surgery (9). Anti-estrogens (e.g. tamoxifen), prostaglandin inhibitors and non-steroidal antiinflammatory drugs (NSAIDs) have also been used. Cytotoxic chemotherapy may be useful in those with disease recurrence or where surgery is contraindicated (10).

\section{CONCLUSION}

Desmoid tumours originate from the musculoaponeurotic structures of the body and can be peripheral or intra-abdominal. Patients should be screened for Gardner's Syndrome and familial adenomatous polyposis coli since these can often present with dermatoid, epidermoid or other benign tumours. Emergency presentations are not common, but are usually secondary to mass effect on the viscera. CT and MRI are the imaging modalities of choice, particularly if surgery is contemplated and also in postoperative follow up to detect recurrence. Surgery is usually the mainstay of treatment but recurrence is common and adjuvant therapy is used. Some tumours possess estrogen receptors, hence they regress in response to anti-estrogens, such as tamoxifen. Postoperative monitoring is essential as recurrence can be as high as $70 \%$.

\section{REFERENCES}

1. Jalini L, Hemming D, Bhattacharya V. Intraabdominal desmoid tumour presenting with perforation. The Surgeon. 2006 Apr; 4(2): 114-6.

2. Schwartz, Robert A, Trovato, Mattew J. Desmoid tumor eMedicine from WebMD. http://www.emedicine.com/derm/ topic778.htm. Last updated: Feb, 2007.

3. Mendenhall WM, Zlotecki RA, Morris CG, et al. Aggressive fibromatosis. Am J Clin Oncol 2005 Apr; 28(2): 211-5.

4. Gonatas NK. Extra-abdominal desmoid tumors. Report of six cases. Arch Pathol 1961Fe; 71: 214-21.

5. Cholongitas E, Koulenti D, Panetsos G, et al. Desmoid tumor presenting as intra-abdominal abscess. Dig Dis Sci 2006 Jan; 51(1): 68-9.

6. Murayama $\mathrm{T}$, Imoto $\mathrm{S}$, Ito $\mathrm{M}$, Matsushita $\mathrm{K}$, Matozaki S, Nakagawa $\mathrm{T}$ et al. Mesenteric fibromatosis presenting as fever of unknown origin. Am J Gastroenterol 1992; 69: 1503-05.

7. Stout AP, Raffale L. Tumours of soft tissues. In: Atlas of Tumour Pathology. Second series. Fascide A. Armed forces Institute of Pathology. Washington DC; 1967.

8. Brueckl WM, Ballhausen WG, Förtsch T, et al. Genetic testing for germline mutations of the APC gene in patients with apparently sporadic desmoid tumors but a family history of colorectal carcinoma. Dis Colon Rectum 2005 Jun; 48(6): 127581.

9. Suit H, Spiro I. Radiation in the multidisciplinary management of desmoid tumors. Front Radiat Ther Oncol 2001:107-19

10. Ohashi T, Shigematsu N, Kameyama K, Kubo A. Tamoxifen for recurrent desmoid tumor of the chest wall. Int J Clin Oncol 2006 Apr; 11(2) :150-2.

Mushtaq Shah, MBBS, MRCS (England) is currently a surgical trainee at Castle Hill Hospital, Cottingham, U.K. He aims to become a general surgeon.

Bushra Azam, MBBS is currently a foundation doctor in Princess Diana Hospital, Grimsby, UK. Her aim is to become a family physician. 